\title{
Quaderni
}

QUADERNI Communication, technologies, pouvoir

97 | Automne 2018

Néo-libéralisme(s). Réseaux et formes des

mobilisations en France

\section{Le capitalisme et ses critiques : l'anti-libéralisme dans la politique française contemporaine}

\section{Émile Chabal}

Traducteur : Kevin Brookes et Constance Péruchot

\section{OpenEdition}

\section{Journals}

\section{Édition électronique}

URL : http://journals.openedition.org/quaderni/1250

DOI : 10.4000/quaderni.1250

ISSN : 2105-2956

\section{Éditeur}

Les éditions de la Maison des sciences de l'Homme

\section{Édition imprimée}

Date de publication : 5 octobre 2018

Pagination : 15-34

\section{Référence électronique}

Émile Chabal, «Le capitalisme et ses critiques : l'anti-libéralisme dans la politique française contemporaine », Quaderni [En ligne], 97 | Automne 2018, mis en ligne le 05 octobre 2020, consulté le 04 janvier 2021. URL : http://journals.openedition.org/quaderni/1250; DOI : https://doi.org/10.4000/ quaderni. 1250 


\section{$D$ ossier}

\section{Le capitalisme}

et ses critiques :

l'anti-libéralisme dans la politique française

\section{contemporaine}

\author{
Émile \\ Chabal
}

Maître de conférences en histoire contemporaine Université d'Édimbourg

Traduction par Kevin Brookes et Constance Péruchot
Durant la majeure partie de son existence, le libéralisme a eu plus d'ennemis que d'amis. À l'exception de quelques brefs moments dans l'Europe de la fin du XIX ${ }^{\mathrm{e}}$ siècle et du début du $\mathrm{XX}^{\mathrm{e}}$ siècle, les libéraux ont rarement dominé la scène politique. Et même lorsqu'ils la dominaient, ils ont été âprement attaqués par tout un éventail d'opposants - des socialistes et communistes, aux fascistes et conservateurs. Parmi toutes les critiques formulées à l'égard du libéralisme, l'une d'entre elles s'est montrée particulièrement efficace, soit l'idée que le libéralisme et le capitalisme ont progressé de concert. Plus récemment, l'émergence du "néolibéralisme» aux États-Unis et au Royaume-Uni à la fin des années 1970 a posé les bases d'une forme agressive de libre-échange capitaliste qui s'est accompagnée d'un regain d'intérêt pour les idées libérales ${ }^{1}$. Pour les antilibéraux de toute part, la proximité des idées radicales des « Chicago Boys » en faveur de l'économie de marché avec les idées libérales radicales de Margaret Thatcher et de Ronald Reagan était indispensable et attendue. L'effondrement du communisme à la fin des années 1980, la généralisation des politiques économiques telles que la «thérapie de choc » ou le programme d'ajustement structurel dans les années 1990 et le triomphe idéologique de la dite « démocratie libérale » ont simplement renforcé le sentiment parmi les antilibéraux que les libéraux montraient de nouveau leur vrai visage, après une longue période passée à contenir les excès du capitalisme au milieu du $\mathrm{XX}^{\mathrm{e}}$ siècle $^{2}$.

Inévitablement, le néolibéralisme agit comme un catalyseur du renouvellement de la politique antilibérale. Tout particulièrement, la relation entre 
le libéralisme et le capitalisme a fait l'objet d'un examen minutieux. Alors que certains aspects du libéralisme de la guerre froide - comme la " démocratie », la « liberté » ou les « droits de l'Homme »- sont parvenus à un certain consensus en Europe de l'Ouest, en Amérique du Nord et au-delà dans les années 1990, il n'en est rien du «capitalisme». Malgré le zèle réformateur d'une nouvelle génération d'économistes néolibéraux, les principes de l'économie de marché n'ont pas engendré le même degré d'approbation à l'ère de l'après-guerre froide. Au moment de la crise financière de 2008, l'anticapitalisme est devenu la forme prééminente de l'antilibéralisme à travers le monde. Il y a peu de meilleurs exemples que la France en la matière. En 2009, l'institut de sondage Globescan a rendu les résultats d'une étude portant sur l'attitude de 27 pays vis-à-vis de l'économie mondiale. Les résultats ont rendu une lecture surprenante : d'après leurs calculs, la France était le pays dans lequel la plus large partie de la population croyait en la nécessité d'un système économique alternatif. Pas moins de $43 \%$ des Français interrogés étaient d'accord avec l'affirmation selon laquelle l'économie de marché " est mauvaise et nous avons besoin d'un autre système ", contre $29 \%$ des Italiens et des Espagnols, $19 \%$ des Anglais, 13\% des Américains et seulement 9\% des Allemands ${ }^{3}$. Cependant, pour le spécialiste de la politique française, la plasticité intrinsèque du discours anticapitaliste contemporain pose des questions méthodologiques complexes. Est-ce un langage protestataire propre à un mouvement politique en particulier? Ou est-ce un marqueur fluctuant - comme le républicanisme - qui change au cours du temps? L'atrophie constante des idéologies majeures structurant la politique française d'après-guerre - communisme et gaullisme en particulier - n'a fait que rendre le sujet plus opaque $^{4}$. La reconfiguration idéologique des années 1970 et 1980 a non seulement rendu les affiliations politiques encore plus difficiles à discerner (tout autant pour l'électorat que pour les politiques eux-mêmes), mais elle a également brisé l'unité du discours anticapitaliste. D'une part, la critique marxiste classique du capitalisme fondée sur la société de classes propre au Parti Communiste Français (PCF) et ses intellectuels alliés sont tombés en désuétude tout comme les idées de « classe » et de « révolution » ont fait l'objet d'un examen intellectuel intense. D'autre part, un anticapitalisme d'inspiration gaulliste basé sur la grandeur nationale et le protectionnisme a fait face à une critique grandissante de la part d'une nouvelle génération d'économistes néolibéraux, hauts fonctionnaires et politiciens cherchant à freiner les excès de l'État technocratique. Dans cet article, je veux examiner un seul aspect de cette « renaissance antibérale », à savoir la reformulation de l'anticapitalisme de gauche dans les années 1980, 1990 et 2000. À première vue, cela peut paraître singulier étant donné les récits traditionnels de la gauche et l'extrême gauche française d'après-guerre qui soulignent, non sans raison, l'écroulement dramatique du marxisme comme force politique et idéologique dans les années $1980^{5}$. Mais ce n'est qu'une partie de l'histoire. Une étude de l'anticapitalisme de gauche révèle que les idées marxistes n'ont pas disparu mais se sont plutôt transformées et fragmentées. Alors que le marxisme lui-même a connu une crise de légitimité, certains de ses concepts structurants - tels que la « classe », la « révolution », la « crise du capitalisme »- ont été repensés afin de corres- 
pondre à une culture politique post-marxiste plus fluide. Surtout, cette transformation d'un langage politique marxiste n'a pas eu lieu dans un contexte de vide idéologique. Au contraire, les anticapitalistes de gauche ont dû tenir compte du néolibéralisme alors même qu'ils le dénonçaient. Comme je le proposerai ici, les nouveaux concepts qu'ils ont fait émerger pour expliquer et critiquer le capitalisme font précisément appel au discours qu'ils essayaient de déconstruire. Cette relation entre la renaissance libérale française et le discours anticapitaliste de gauche n'était donc pas celle d'une simple hostilité mutuelle mais bien celle d'une symbiose et d'un dialogue.

Cela est particulièrement visible si nous nous concentrons sur le degré de la théorie, ce pourquoi j'ai choisi d'examiner l'éventail du discours anticapitaliste en France à travers quatre textes emblématiques : Adieux au prolétariat : au-delà du socialisme d'André Gorz (1980), l'essai « République ou Démocratie » de Régis Debray (1989), Le nouvel esprit du capitalisme de Luc Boltanski et Ève Chiapello (1999), La nouvelle raison $d u$ monde : essai sur la société néolibérale de Pierre Dardot et Christian Laval (2009). D'une certaine manière, ces textes sont très dissemblables : ils ont été écrits par des universitaires et intellectuels de disciplines variées et ils adoptent des approches radicalement différentes de la critique du capitalisme. Ils n'ont pas non plus le même format puisque l'un est un essai court, un autre est long et deux d'entre-eux sont des monographies universitaires complètes. Pourtant, malgré leurs différences, ces quatre textes apportent la base d'un corpus cohérent sur lequel fonder une analyse préliminaire de l'évolution et la diversité de l'anticapitalisme français contemporain. Ils ont relativement tous eu du succès en France lors de leur publication et ils ont provoqué un débat bien au-delà du monde académique, particulièrement dans les cercles activistes, politiques et les syndicats. De plus, et ceci est essentiel, leurs auteurs se sont tous considérés comme étant à gauche (même s'ils n'étaient pas d'accord avec la teneur de cette " gauche »). Cela signifie que, malgré leurs approches opposées à ce propos, ils ont tous fait appel aux mêmes cadres (souvent à partir d'une variété post-marxiste), ont partagé un répertoire de concepts et se sont occasionnellement référés les uns aux autres. Dans un court chapitre, il est impossible d'explorer en détail les complexités de chaque texte. Mon attention principale se portera plutôt sur la manière dont les quatre textes conceptualisent le pouvoir dans un monde plus incertain et fragmenté 6 . Alors que le néolibéralisme est devenu une idéologie globale dominante dans les années 1980 et 1990, ses critiques ont discrédité les notions structurelles rigides de « classe », « prolétariat » ou « classe dominante » pour les remplacer avec des idées plus souples du pouvoir telles que l'« esprit» ou la « rationalité » du capitalisme, qui étaient plus adaptées à une époque de mondialisation rapide. Chacun des quatre textes tente d'isoler les sources instables et décentralisées toujours plus nombreuses du pouvoir dans un monde néolibéral et proposent des alternatives, de l'écologie politique à une régulation étatique des marchés. L'éventail des solutions reflète à la fois la diversité des généalogies intellectuelles en jeu dans chaque texte, mais également la tendance à l'atomisation du néolibéralisme lui-même. Dans un monde de pluralisme par le marché et de démocratie individualiste, les penseurs critiques de gauche ont d'abord dû identifier qui ou ce 
qui tenait réellement les rênes du pouvoir afin de suggérer comment un tel pouvoir peut être modifié, limité ou démantelé totalement.

\section{L'écologie politique et la fin du capitalisme}

André Gorz publie son essai Adieux au prolétariat : au-delà du socialisme en 1980, peu de temps avant la victoire socialiste mémorable en 1981. Gorz était alors déjà un penseur important parmi la gauche dissidente non-communiste et une partie du mouvement syndical - notamment la Confédération Française Démocratique du Travail (CFDT) menée par Edmond Maire - et il avait publié des ouvrages de philosophie et de politique depuis la fin des années 1950. Néanmoins, sa fusion inattendue entre l'existentialisme sartrien et « l'écologie politique » l'a fermement positionné en dehors de la gauche française traditionnelle ; bien qu'il ait été proche de Sartre et l'éditeur de la revue Les Temps Modernes de 1961 jusqu'au milieu des années 1970, il s'est progressivement détaché de ce milieu. Il était aussi un homme particulièrement modeste : il était tellement attaché à son anonymat qu'il utilisait différents pseudonymes - André Gorz pour ses écrits philosophiques et Michel Bosquet pour ses écrits journalistiques (d'abord pour L'Express, puis ensuite en tant que fondateur et rédacteur en chef du Nouvel Observateur à partir de 1964) ${ }^{7}$. Contrairement à l'image de l'affirmation autonome de l'intellectuel français d'après-guerre, Gorz était un philosophe universitaire et un journaliste, très engagé dans la critique du capitalisme, qu'il a développée sans relâche au cours de plusieurs décennies ${ }^{8}$. Le succès de Adieux au prolétariat était donc quelque peu surprenant. Son but était de déclencher un débat sur la valeur du travail salarié et les contradictions du capitalisme, des questions à propos desquelles Gorz avait déjà largement écrit durant la décennie précédente ${ }^{9}$. Mais la véritable perspective d'une victoire de l'union de la gauche socialiste et communiste à la prochaine élection et les fortes atteintes au marxisme durant la vague anti-totalitaire de la fin des années 1970 a soudainement préparé le terrain pour la critique radicale et non-communiste de Gorz $^{10}$. Le titre du livre, qui proclame la fin de la classe ouvrière comme entité cohérente et révolutionnaire, a causé une controverse majeure parmi le mouvement syndical dévoué à la notion de lutte des classes, mais il a su percevoir quelque chose d'essentiel à propos de la réalité socioéconomique changeante en France après la fin de l'essor économique d'après-guerre. Il n'est pas anodin que Gorz ait choisi d'inclure en annexe du livre une analyse des données d'une récente enquête suggérant que les valeurs politiques et sociales devenaient de plus en plus détachées de la classe socioéconomique. Comme il l'indique : «Il est (...) nécessaire de reconnaître l'existence d'une crise culturelle, l'émergence d'une nouvelle sensibilité et de nouvelles valeurs qui, transcendant les anciennes frontières entre classes, ne trouvent pas toujours d'expression politique appropriée ${ }^{11}$. » En somme, le socialisme avait besoin de développer de nouvelles bases et solutions afin de conserver son attrait dans un contexte socioéconomique plus fluide. Il s'agissait précisément du but de Gorz dans Adieux au prolétariat. Le livre a commencé par exposer une critique virulente de la notion marxiste de « prolétariat ». C'était, selon Gorz, à la fois philosophiquement et empiriquement problématique. Philosophiquement car pour que la révolution 
communiste ait lieu, la classe ouvrière devrait se nier en renonçant au travail salarié qui formait empiriquement la base de son identité, parce qu'il $\mathrm{y}$ avait de moins en moins de preuve que tout ce qui ressemble au prolétariat puisse exister dans des sociétés capitalistes flexibles et avancées. Les tentatives de plus en plus désespérées de la part des mouvements socialiste et communiste pour « identifier » et parler « pour le compte » du prolétariat étaient par conséquent vouées à l'échec. De même, la perspective d'un « pouvoir de l'ouvrier » ou d'une « dictature du prolétariat » n'était rien de plus qu'une chimère ; le prolétariat, s'il a jamais existé, était encore moins susceptible de devenir une réalité. Gorz expliquait que le réel problème du capitalisme n'était pas sa structure de classe mais sa fétichisation du travail salarié et sa vision utilitariste du travail. La gauche devait se concentrer, non pas sur la libération d'un prolétariat fictif, mais sur « l'abolition » d'une conception capitaliste du travail et son remplacement par une attitude plus holiste et épanouissante du travail, d'autant plus que les nouvelles technologies menaçaient de rendre le travail salarié obsolète. Dans ce futur socialiste - que Gorz a volontiers appelé une « utopie »-le travail serait récompensé pour son utilité sociale plutôt que rémunéré par des salaires. En retour, cela casserait les mécanismes d'accumulation capitaliste qui détruisaient progressivement la biodiversité de la planète et aliénaient ses habitants.

Le texte de Gorz semble remarquablement prescient $^{12}$. Déjà à cette époque, une grande partie de la classe politique de France était consciente que le repli économique et le chômage étaient devenus la norme, et rien n'a vraiment changé depuis $\operatorname{lors}^{13}$. L'équation systématique entre l'accumulation capitaliste et la dégradation environnementale - désormais connue sous le nom d' « écologie politique »- est devenue une évidence parmi les mouvements écologistes en Europe $^{14}$. Et surtout, la conception du pouvoir dans les économies capitalistes par Gorz est celle que de nombreux critiques de gauche du capitalisme reconnaitraient aujourd'hui. Dans Adieux au prolétariat, Gorz pose son argument explicitement : dans une économie capitaliste avancée, le pouvoir n'est pas conféré à une classe dominante omnipotente ; au contraire le « secret de la production industrielle de masse (...) est que personne n'y détient le pouvoir ${ }^{15}$. » Le pouvoir est diffusé à travers le système économique, les rangs inférieurs étant tout autant impliqués que les rangs élevés. C'est pourquoi il est insensé de dire que le prolétariat devrait réclamer le pouvoir, puisqu'en faisant cela il ne ferait que reproduire les inégalités systématiques du capitalisme. Pire encore, quels que soient les échos d'un prolétariat qui aurait pu exister au début et milieu du $\mathrm{XX}^{\mathrm{e}}$ siècle, ils sont rapidement remplacés par ce que Gorz appelle une «non-classe des prolétaires post-industriels ${ }^{16}$. » Ces personnes sans emploi ou intérimaires sont superflues pour le développement économique ; elles ont en effet été « écartées » par le capitalisme. Parce qu'elles vivent à présent aux marges de la société, elles n'ont « aucune conception de la société à venir (...) nul messianisme, nulle théorie globale [n'assurant] sa cohésion et la continuité de son action $^{17}$.» Encore une fois, l'opinion de Gorz était un signe des changements à venir : aujourd'hui la plupart des critiques français de gauche acceptent l'idée d'une sous-classe d' " exclus » et de « marginalisés ». La différence était alors que, dans Adieux au prolétariat, Gorz estimait 
que les membres de cette « non-classe » étaient l'avant-garde du changement révolutionnaire. Libérés des fers du capitalisme salarié, ils étaient à présent libres de développer une conception plus épanouissante et non-salariale du travail. C'est pourquoi Gorz défendait un salaire décent garanti et une réduction des heures de travail : ces mesures permettraient d'étendre les logiques du travail non-salarié à toute la main-d'œuvre. Si les humains avaient assez pour vivre, déclare Gorz, ils se détourneraient progressivement du travail salarié et se consacreraient à des activités de « loisir » productives.

Rétrospectivement, il est clair que Gorz surestime les contradictions du travail salarié capitaliste et le potentiel révolutionnaire de sa «non-classe ». Il avait indubitablement raison de dire que le capitalisme basé sur la technologie de pointe crée une main-d'œuvre temporaire plus instable, mais en France, cette tranche est devenue de plus en plus associée à la montée du Front National à l'extrême droite plutôt qu'aux politiques révolutionnaires de gauche ${ }^{18}$. Néanmoins, le plus intéressant est bien que cet aspect de l'argument de Gorz a déplacé le pouvoir des catégories collectives de l'analyse telles que la « classe » vers l'individu. Le changement révolutionnaire était systématique - dans le sens où il était cumulatif - mais mené par des acteurs individuels. En valorisant l'importance de l'épanouissement personnel, il insistait sur les rôles clés que les individus pouvaient jouer dans leur propre émancipation. Le résultat, dans une économie capitaliste avancée où personne ne détient réellement le pouvoir, était l'instigation du changement révolutionnaire à travers la construction d'une multitude d'espaces « non-capitalistes » autonomes et individuels.
Il a explicitement rejeté l'idée d'un isolement individuel - personne ne pourrait simplement « quitter » la société - mais il maintenait que les espaces autonomes étaient nécessaires afin d'encourager les « expérimentations sociales » et de construire un système socioéconomique durable qui serait davantage en harmonie avec $l^{\prime}$ 'environnement nature ${ }^{19}$. Il a tenu cette position jusqu'à sa mort en 2007 : pour Gorz, les changements du marché du travail, qui ont mené à la création d'une immense " non-classe », étaient à la fois un symptôme de la crise du capitalisme et également les moyens par lesquels la société humaine pourraient enfin engager sa « sortie du capitalisme $^{20}$. » L'accent mis sur l'émancipation de l'individu et l'épanouissement personnel dans Adieux au prolétariat s'explique de manière évidente connaissant l'intérêt de longue date de Gorz pour l'aliénation et les premiers travaux de Marx, mais je pense qu'il est significatif que ses idées aient attiré l'attention d'un plus large public au début des années 1980. L'une des raisons réside dans le fait que ses thèses se sont heurtées à deux des approches de la réforme du capitalisme dominantes à gauche à l'époque : une théorie communiste pure de la lutte des classes et l'idée du contrôle des travailleurs et leur auto-gestion, qui était à la mode parmi les membres de la deuxième gauche à la fin des années $1970^{21}$. Mais c'était aussi parce que les grandes lignes de Gorz font sens pour une jeune génération intéressée par les nouveaux mouvements sociaux, la politique identitaire et le droit à la différence, et qui a cherché à créer des espaces favorables à l'autonomie de l'individu au-delà de la « portée » de l'accumulation capitaliste. Plus généralement, les arguments de Gorz reflètent ceux du néolibéralisme : ce dernier accentue éga- 
lement l'importance de l'autonomie individuelle et la diffusion du pouvoir (dans ce cas, grâce à la «main invisible» du marché). Même s'ils ne parviennent pas aux mêmes conclusions à propos de la valeur du système capitaliste, ils partagent une vision similaire de la société contemporaine et l'essoufflement des idéologies collectives. Sur la longueur, le néolibéralisme serait le plus influent des deux, mais la puissance de la critique de Gorz réside dans le fait qu'elle semble s'articuler avec, et non pas contre, la configuration idéologique et socioéconomique émergente des années 1980.

\section{Que vive la République !}

La différence entre Adieux au prolétariat et le fameux essai de Régis Debray « République ou Démocratie » (1989) est tellement importante que l'on peut raisonnablement se demander s'il y a même un lien entre eux ${ }^{22}$. L'essai de Debray ne cite le mot de « capitalisme » qu'une seule fois, il n'inclut aucune discussion soutenue sur le travail salarié ou la société postindustrielle et il ne fait référence à aucun autre auteur ou texte. Pourtant, je dirais que sa conceptualisation du pouvoir et son argumentation fortement néorépublicaine à propos de l'incompatibilité entre une "République » et une " Démocratie » le positionne fermement au sein d'une tradition stylistique de gauche et anticapitaliste. En effet, les contrastes entre les textes de Gorz et Debray offrent un aperçu utile de la diversité de la pensée anticapitaliste en France. Ainsi, là où Gorz affirme que le pouvoir est diffusé à travers le système industriel, Debray soutient que le pouvoir repose sur les médias et « l'image »; et, là où Gorz défend l'émancipation individuelle par le travail, Debray maintient qu'un État rationnel et républicain est la seule manière de transcender les carences du capitalisme néolibéral.

À la fin des années 1980, Debray était déjà un philosophe et un intellectuel reconnu. Il avait passé quelque temps en Amérique latine en tant que membre du mouvement de guérilla de Che Guevara au milieu des années 1960 et il avait été l'un des conseillers de François Mitterrand au début des années 1980. Il a plus particulièrement acquis une notoriété pour sa critique des protestations de 1968 (durant lesquelles il était en prison en Amérique latine) et pour son engagement ultérieur aux orientations étatistes assumées des années Mitterrand. Mais il a fallu un contexte particulier pour que Debray mette par écrit son républicanisme, à savoir la controverse entourant l'expulsion de trois jeunes filles qui portaient le voile dans un collège public à Creil en septembre 1989. En quelques semaines, ladite affaire du foulard est passée d'un problème local à une célèbre cause nationale. Des personnalités publiques de tout bord ont montré leur soutien au directeur qui a exclu la jeune fille au motif qu'il faisait respecter les « valeurs » de la République française, alors que d'autres ont dénoncé cette mesure comme étant un nouvel exemple de «l'intolérance» française ${ }^{23}$. Parmi la cacophonie des opinions, Debray est devenu l'une des voix parmi les plus prééminentes (et polémiques). Il s'est fermement rallié au directeur et il a écrit un certain nombre de textes qui défendaient l'intégrité, la moralité et l'applicabilité des valeurs « républicaines ${ }^{24}$. » L'un des plus connus est «République ou Démocratie », qui a été publié sous la forme d'un long article dans $L e$ Nouvel Observateur en novembre 1989. Dans ce texte, le cœur de l'argument de Debray est 
la distinction normative profonde à effectuer entre une « République » et une « Démocratie» (il met une majuscule aux deux termes) ${ }^{25}$. Une République correspond à l'incarnation publique du rationalisme éclairé, alors que la Démocratie est simplement l'expression de l'opinion collective de la société. Selon ses mots : «La République est la Démocratie plus. La République, c'est la liberté, plus la raison. L'État de droit, plus la justice. La tolérance, plus la volonté. La Démocratie (...) c'est ce qui reste d'une République quand on éteint les Lumières ${ }^{26}$. »

Mais Debray fait bien plus que construire une comparaison normative entre la République et la Démocratie ; il établit également une longue liste d'associations qui donnent une vision plus nette de ses propres idées préconçues. Dans ce contexte, il faut particulièrement nous intéresser à ses discussions sur le soi-disant lien de la Démocratie avec le commerce, la publicité et les médias. Quelques exemples suffiront à donner une idée de son argument. Dans une Démocratie, "l'opinion fait loi », "l'argent est maître de tout » et «le client est ro $i^{27}$. » Cela fait écho aux personnalités clés d'une Démocratie : «les juristes (...) le journaliste, le publicitaire, le chanteur, l'acteur, l'homme d'affaires ${ }^{28}$. $)$ Surtout, la Démocratie a différentes priorités. Alors que la République se concentre de manière obsessionnelle sur " l'institution », une Démocratie est en fin de compte uniquement concernée par « la communication $^{29}$. » Debray reconnaît bien que toutes les démocraties ne sont pas aussi clairement libérales ou consuméristes qu'il le souhaite, mais il soutient que dans la période actuelle, « la société libérale et consumériste [est] si dominante et communicative qu'on la croit obligatoir ${ }^{30}$. »
Dans son analyse, la démocratie, le libéralisme et la société de consommation sont donc tous interdépendants. L'opposition binaire caricaturale de Debray entre une République (française) et une Démocratie (américaine) se rapproche dangereusement des pires traits de l'antiaméricanisme primaire de la France ${ }^{31}$. Mais si nous laissons de côté cette critique, nous pouvons mieux observer les similitudes entre l'anticapitalisme de Debray et celui des autres auteurs dans ce chapitre. Ceci est particulièrement visible dans sa conceptualisation du pouvoir. Comme dans le cas de Gorz, Debray ne considère pas le pouvoir comme étant attribué à une classe spécifique mais plutôt une valeur dominante qui privilégie le baratin, les images superficiels et le succès financier. Comme il l'écrit sans ménagement : "L'idéal républicain postule (...) un certain respect des proportions. Les salaires faramineux des vedettes et des puissants du jour, par hasard révélés au public, ne suscitent chez le fauché démocrate qu'un haussement d'épaules, simples rançons, dira-t-il, de la liberté d'entreprendre ${ }^{32}$. »

Pour Debray, la question de savoir si l'inégalité des salaires est bonne ou mauvaise ne se pose pas dans une Démocratie - seulement la question de sa perception. Alors que les Républiques rejetteraient l'idée d'une accumulation illimitée, il n'existe pas de telle limite dans une Démocratie. Au contraire, «les meilleurs dans une République vont au prétoire et au forum », tandis que «les meilleurs en Démocratie font des affaires ${ }^{33} . » \mathrm{Le}$ pouvoir dans une Démocratie repose alors sur ceux qui sont capables de négocier des valeurs capitalistes avec succès. Il est clair pour Debray que ces valeurs ont été de plus en plus transmises à travers les médias américains qui privilégient 
les images aux idées et la publicité au contenu ${ }^{34}$. Depuis la fin des années 1970, Debray s'est intéressé à la relation entre les images et l'idéologie - ce qu'il appelle la « médiologie » - et dans « République ou Démocratie », il utilise une version simplifiée de sa théorie dans le but de condamner l'impact de la mondialisation sur la culture $^{35}$. Dans le texte, Debray décrit de manière frappante la désorientation entraînée par la culture américaine : selon lui, cela a rendu la culture de soi (c'est-à-dire la culture française) étrangère, et la culture de l'Autre (c'est-à-dire la culture américaine) comme faisant partie de soi $^{36}$. La manipulation du concept marxiste d'aliénation fait remarquablement écho à Gorz, sauf lorsque Gorz se concentre sur le travail salarié comme l'aspect le plus corrosif du capitalisme, là où Debray se concentre sur la production culturelle. « L'appauvrissement » culturel de la France est une conséquence directe de la démocratie et de la fascination du capitalisme pour le style plutôt que le fond. Au fil du temps, cela aliène les citoyens des racines civiques de la citoyenneté, brise les liens de la solidarité entre eux et enfin, les rend impuissants face à la plus pure des cultures capitalistes, celle des États-Unis. Mais si l'adaptation de l'aliénation a un air de Gorz, la solution proposée est loin de l'idée d'espaces autonomes, individuels et non-capitalistes. Au contraire : Debray est ambigu à propos de la nécessité d'une autorité centrale rationnelle forte sous la forme d'une République. En tant que recueil de la mémoire nationale, le patrimoine culturel et les valeurs des Lumières, la République est le seul rempart possible contre la décadence de la Démoratie et les valeurs financières du capitalisme. Durant la décennie précédente, c'était «l'État et ses censures qui menaçait l'autonomie de l'individu » mais « aujourd'hui c'est la «société civile» (...) et la montée de pouvoirs de plus en plus intolérants et incontrôlés - médias, clergés, sciences, administration ». Paradoxalement, la seule manière de préserver « l'autonomie individuelle » passe par une défense intransigeante de la République (française) et de ses valeurs ${ }^{37}$. Pour Debray, cette émancipation est possible à travers la République et ses institutions séculaires. Cette défense ouvertement étatique et néorépublicaine de la République a été assez légitimement critiquée pour son élitisme et son ethnocentrisme ${ }^{38}$. Mais Debray n'était pas l'ancien seul ex-radical de gauche à s'être tourné vers la République dans les années 1980 et 1990 . D'autres penseurs tels que Alain Finkielkraut et Blandine Kriegel ont suivi la même réflexion et ont appuyé des arguments similaires la plupart du temps ${ }^{39}$. Ils ont encore et encore attaqué le pouvoir insidieux des valeurs capitalistes et insisté sur le pouvoir d'émancipation de la République séculaire. De plus, comme la controverse du foulard a traîné en longueur jusqu'à la loi de 2004 bannissant les signes religieux à l'école, il y avait beaucoup d'opportunités pour les intellectuels néorépublicains de se faire entendre dans la sphère publique. Au milieu des années 2000, une palette de politiciens et d'intellectuels publics ont invoqué la République comme défense contre « le multiculturalisme », « le communautarisme », « le capitalisme anglosaxon » et « l'ultralibéralisme » - une tendance dont l'expression politique la plus claire a été le vote $\mathrm{du}$ « Non » dans le référendum sur la Constitution européenne en $2005^{40}$. Pour toutes ses qualités polémiques, l'essai de Debray de 1989 a signalé l'arrivée d'un nouveau discours anticapitaliste, qui exerce encore une emprise considérable sur la politique française. 


\section{Les esprits du capitalisme?}

La dense étude de 800 pages rédigée par Luc Boltanski et Ève Chiapello, intitulée Le nouvel esprit du capitalisme (1999), était distincte des deux précédents textes en ce qu'il a essayé d'expliquer, non pas simplement ce qui n'allait pas avec le capitalisme avancé, mais aussi la raison pour laquelle les critiques contemporains du néolibéralisme ont échoué à avoir beaucoup d'impact ${ }^{41}$. Ce n'est pas surprenant, étant donné que le livre a été écrit au milieu des années 1990, alors que tous les espoirs d'une critique acerbe du néolibéralisme venant de la gauche semblaient s'être évaporés. Sur le plan international, la chute du Mur de Berlin et la fin du communisme en Europe a confirmé la « victoire » retentissante du libéralisme et de la démocratie ; et au niveau national, la défaite de Jospin (PS) à l'élection présidentielle de 1995 a marqué la fin d'une période longue et difficile de gouvernance socialiste, durant laquelle de nombreuses valeurs chéries par la gauche française ont été mises de côté au nom de l'austérité et du pragmatisme ${ }^{42}$. Il fallait désormais expliquer la persistance de l'ordre néolibéral. Cela impliquait d'identifier et de conceptualiser le pouvoir, et ainsi les arguments principaux de l'ouvrage sont connectés avec ceux de Gorz et Debray. Il semblait plus que jamais urgent d'offrir une théorie de la manière dont le capitalisme est durable en lui-même ou, pour reprendre les termes que les auteurs eux-mêmes ont utilisé, la manière dont le capitalisme a réussi à « se justifier » lui-même ${ }^{43}$.

Ce vaste projet a puisé dans les écrits antérieurs des deux auteurs. Boltanski a travaillé avec Pierre
Bourdieu au Centre de Sociologie des Organisations dans les années 1970 et ils ont publié un certain nombre de volumes en collaboration. Les deux intellectuels ont fini par se séparer intellectuellement, Boltanski considérant que la conception bourdieusienne de la « sociologie critique » n'était pas suffisamment subtile et pragmatique pour prendre en compte le vaste champ des motivations qui ont poussé les acteurs individuels. Néanmoins, Boltanski a conservé l'intérêt de Bourdieu pour la formation d'un groupe identifié, la sociologie organisationnelle et la stratification sociale. Chiapello est quant à elle issue d'un parcours en théorie de la gestion. Elle a enseigné (et enseigne encore) à l'école des Hautes Études Commerciales de Paris (HEC) et avait précédemment écrit à propos de l'émergence d'une nouvelle classe managériale. Cela lui a permis d'avoir une vision perçante de la structure organisationnelle du capitalisme qui, combinée à l'intérêt de Boltanski pour les approches critiques de l'idéologie, a donné au Nouvel esprit du capitalisme une base théorique puissante. Cela repose surtout sur l'idée selon laquelle il y aurait eu des « esprits » successifs du capitalisme depuis le dix-neuvième siècle, chacun ayant fourni des justifications et des idées appuyant la légitimité du système économique existant. Le « nouvel» esprit correspond alors à la forme la plus récente du capitalisme : le capitalisme néolibéral des années 1980.

Les auteurs développent leur argument en trois parties. Premièrement, à travers une analyse du discours des manuels de gestion des années 1960 aux années 1990 afin de montrer comment l'image idéale du manager - et par extension, du capitalisme dans son ensemble - a changé durant 
cette période. Dans les années 1960, l'accent était mis sur des objectifs clairs, l'avancement professionnel et la hiérarchie bienveillante ; dans les années 1990, les manuels de gestion clamaient la valeur de la flexibilité, la diversité des projets et les relations « réseautées » entre individus et entreprises ${ }^{44}$. Deuxièmement, les auteurs explorent les façons dont la « critique artistique » du capitalisme, qui a atteint son apogée en 1968, a été par la suite admise et absorbée dans le discours dominant du capitalisme néolibéral à partir des années 1970. Ce glissement s'est produit en parallèle d'une transformation du marché du travail ayant brisé les unités collectives d'organisation telles que les syndicats, qui étaient jusqu'ici les dépositaires d'une « critique sociale » du capitalisme. Il en est résulté que toutes les formes de critique ont été désarmées dans les années 199045. Enfin, les auteurs se concentrent sur les possibilités renouvelées de la critique. En particulier, ils suggèrent que les effets néfastes du capitalisme néolibéral sur les populations marginalisées et exclues pourraient constituer la base d'une nouvelle catégorie « d'indignation morale ». Cette idée se manifeste alors dans les nouvelles formes de justice et de législation de l'État pour protéger les plus vulnérables.

Les arguments de Boltanski et Chiapello ont été largement repris en France et dans le monde anglo-saxon (une fois que le livre a été traduit en anglais en 2005) ${ }^{46}$. De toute évidence il s'agit d'un succès majeur dans le domaine de la sociologie critique, mais le plus intéressant pour notre propos est la manière dont cela a étendu et développé une critique française du capitalisme et du néolibéralisme qui était déjà visible dans les deux précédents textes de Gorz et Debray. Les liens avec le premier sont les plus évidents. Boltanski et Chiapello consacrent un certain nombre de pages à la disparition de l'idée de « classe sociale » et de « l'affaiblissement des défenses du travail », chacun des deux étant au cœur du travail de Gorz à partir des années $1980^{47}$. De même, l'idée de l'épanouissement par l'autonomie est présente dans Le nouvel esprit du capitalisme, sauf que là où Gorz y voit la possibilité d'une " sortie du capitalisme », c'est l'inverse pour Boltanski et Chiapello, qui affirment que l'aspiration à l'autonomie a été entièrement intégrée par la logique du capitalisme néolibéral basé sur le réseau ${ }^{48}$. L'appel de Gorz à la construction d'espaces autonomes non-capitalistes n'est alors pas adéquat étant donné que le capitalisme néolibéral a adapté l'idée d'autonomie pour correspondre à son propos. La seule solution adéquate est de se tourner vers l'État comme source de régulation et législation. C'est ici que le lien avec le néorépublicanisme de Debray intervient. Il n'y a aucune mention d'une République transcendantale dans l'argumentaire de Boltanski et Chiapello, mais l'État joue un rôle crucial pour définir « la place de la loi » et construire des « nouveaux systèmes de justice » qui peuvent limiter les effets abominables du capitalisme néolibéral ${ }^{49}$. Selon leurs mots : «La possibilité pour le capitalisme de s'auto-contraindre ne dépend pas seulement de la force de la critique mais aussi de la force des États sur lesquels le capitalisme doit compter pour que ceux qui en assurent le fonctionnement se trouvent liés à leur promesse $d$ 'autolimitation et respectent ce qui, dans les contrats, concerne non seulement les intérêts des parties mais aussi le bien commun ${ }^{50}$. » Un peu plus loin, les auteurs affirment que : «Une politique publique responsable [peut] faciliter 
(...) la critique, en permettant la représentation dans le débat politique des personnes souffrant le plus ${ }^{51}$.»

Cet appel retentissant adressé à l'État pour donner la parole aux plus vulnérables et intervenir afin de contraindre les entreprises à respecter les injonctions morales du nouvel esprit du capitalisme suppose que l'État est une force à la fois bienveillante et rationnelle dans la société. Une telle vision serait totalement en accord avec la conception néorépublicaine de l'État de Debray. Compte tenu du fait que Boltanski et Chiapello ne proposent aucune théorie de l'État - et ne discutent pas des effets du nouvel esprit du capitalisme sur l'État et les institutions du secteur public - l'on se retrouve avec une vision résolument néo-républicaine de la légitimité de l'État et de l'action de l'État. De plus, la discussion complexe de Boltanski et Chiapello sur la manière dont la « critique artistique » de 1968 est devenue partie intégrante du nouvel esprit du capitalisme fait écho à l'affirmation polémique de Debray selon laquelle une élite soixante-huitarde politiquement correcte s'est laissé tenter par le triptyque dominant Démocratie-Libéralisme-Capitalisme. Dans la postface de leur édition de 2010, Boltanski et Chiapello ont reconnu qu'ils avaient surestimé la capacité de l'État à agir comme un rempart contre le capitalisme, et exagéré la relation entre «l'esprit artistique » de 1968 et le nouvel esprit du capitalisme ${ }^{52}$. Et Boltanski lui-même en 2011 a spécifiquement attaqué les néorépublicains pour s'être cramponnés à une vision de l'État « largement illusoire ${ }^{53}$. » Ces désaveux rétrospectifs n'ont cependant pas éliminé l'étude analytique du texte original, qui a été écrit entre 1995 et 1999. Au même moment, les critiques de gauche du capitalisme et du néolibéralisme ont cherché de nouvelles manières de formuler une critique aiguisée du système économique dans lequel ils vivaient. Pour ce faire, ils avaient besoin d'une théorie du pouvoir du capitalisme plus appropriée que celles proposées par Gorz (la reproduction de normes capitalistes à travers la division industrielle du travail) ou Debray (la propagation des valeurs capitalistes à travers les médias). Le grand avantage de la notion d'« esprit» de Boltanski et Chiapello - empruntée au travail de Max Weber - était qu'elle répondait clairement à la manière dont la configuration économique absorbait les arguments et ses détracteurs. Leur définition minimale du capitalisme comme « exigence d'accumulation illimitée du capital par des moyens formellement pacifiques » signifiait que le capitalisme pourrait facilement s'adapter à n'importe quel cadre idéologique existant ${ }^{54}$. Dans un contexte où les frontières entre la droite et la gauche en France devenaient de plus en plus floues dans les années 1990 - et où aucun gouvernement ne semblait capable d'une réforme économique - cette vision était attirante pour la gauche anticapitaliste ${ }^{55}$. Surtout, l'argument de Boltanski et Chiapello semblait reconnaître la fragmentation croissante du pouvoir dans un monde néolibéral et la difficulté concomitante à construire une critique efficace et focalisée.

\section{Nous sommes tous des néolibéraux}

Le livre de Pierre Dardot et Christian Laval, La nouvelle raison du monde : essai sur la société néolibérale (2009), a été inspirée par une publication en 2004 des cours de Michel Foucault sur la «biopolitique », qu'il a donnés en 1978-197956. La mise à disposition de ces nouveaux textes a 
été un événement important pour les antilibéraux français étant donné que les cours semblent montrer, plus clairement que jamais avant, que Foucault a développé une critique cohérente du néolibéralisme en tant que « gouvernementalité ». Cela implique que le néolibéralisme n'est pas simplement une idéologie dominante qui trouve continuellement de nouvelles manières de se justifier, mais plutôt qu'il gouverne les actions individuelles et la rationalité intérieure de ceux qui vivent dans son ombre. Comme certains historiens des idées l'ont fait remarquer, cette lecture de Foucault comme critique précoce du néolibéralisme est, au mieux, une interprétation partielle de ses écrits ultérieurs ${ }^{57}$. Mais cela n'a pas empêché Dardot et Laval de l'invoquer comme base conceptuel de leur ouvrage. Selon eux, les connaissances théoriques de Foucault ont permis de développer un argument à propos de la source du pouvoir néolibéral, qui est à la fois individuelle et globale. En effet, la « rationalité » néolibérale de Dardot et Laval est tellement large que même la crise financière catastrophique de 2008 et les crises de la dette souveraine ultérieures ne suffisent pas à la détruire : nous pouvons échapper au néolibéralisme seulement à partir du moment où nous réalisons que nous sommes sous son emprise. Cette vision assez morne trouve ses origines dans les intérêts politiques et intellectuels des deux auteurs. Laval est un professeur de sociologie au Laboratoire Sophiapol de l'Université Paris Ouest-Nanterre. Il a commencé sa carrière dans les années 1990 en travaillant sur l'histoire de l'utilitarisme - plus particulièrement sur la vie et le travail de Jérémy Bentham $^{58}$. En parallèle de son travail sur le dix-neuvième siècle, il a commencé à s'engager plus étroitement dans les débats contemporains sur la gauche française en tant qu'intellectuel et activiste d'extrême-gauche. En 2004, il a publié une attaque virulente de la néolibéralisation insidieuse du système d'école publique, et la même année, il a créé le groupe d'études Question Marx, dont le but assumé était de créer un forum pour l'élaboration d'une nouvelle pensée critique marxiste ${ }^{59}$. C'est au sein de cette structure que Laval a commencé à travailler sur de nombreux projets collectifs avec Dardot - un philosophe sans affiliation académique formelle ${ }^{60}$. Ils ont désormais publié un certain nombre de textes collaboratifs sur la pensée critique, Marx et le marxisme, et l'idée de révolution, mais celui qui a eu le plus grand impact dans le cercle des activistes français de gauche a été La nouvelle raison $d u$ monde, qui cherchait à combiner une approche « historique » du néolibéralisme et une critique de ses manifestations contemporaines.

Les auteurs présentent leur intention dès la première page. Ce n'est pas un livre à propos de l'idéologie ou les bases socioéconomiques du néolibéralisme, mais plutôt sur le mode de vie néolibéral : "Nous n'en avons pas fini avec le néolibéralisme. Quoi qu'en pensent beaucoup, ce dernier n'est pas une idéologie passagère (...) il n'est pas seulement une politique économique (...) il s'agit de bien autre chose, il s'agit de bien plus; de la manière dont nous vivons, dont nous sentons, dont nous pensons. Ce qui est en jeu n'est ni plus ni moins que la forme de notre existence $(\ldots)^{61}$. »

Le but de cette introduction véhémente est de souligner le fait que le néolibéralisme est devenu à la fois une technologie du pouvoir et une nouvelle forme de rationalité globale. Pour Dardot 
et Laval, le capitalisme constitue une part intégrante du néolibéralisme, mais celui-ci ne peut être réduit à un système économique. Au lieu de cela, les deux auteurs choisissent de se concentrer sur la création d'une « subjectivité » libérale à travers l'histoire - du libéralisme classique du dix-huitième et dix-neuvième siècle au néolibéralisme agressif de la fin du vingtième siècle. Cela requiert une longue discussion historique et les premiers chapitres sont consacrés à une série d'auteurs, tels que Bentham, Spencer, Röpke, Hayek et Friedman, dont les écrits sont considérés comme étant essentiels aux différentes étapes du libéralisme. À cela s'ajoute une focalisation sur des institutions et mouvements particuliers, particulièrement le colloque Walter Lippmann et l'émergence de l'ordolibéralisme dans les années 1930 et 1940. Ce vaste historique donne les clés de la compréhension des bases de la subjectivité néolibérale.

Les historiens des idées ont soulevé des questions légitimes à propos de la lecture décontextualisée et téléologique de la tradition libérale occidentale par Dardot et Laval ${ }^{62}$. La troisième partie du livre est cependant encore plus pertinente, en ce que les auteurs y attirent l'attention sur le « grand point tournant » des années 1980 et la généralisation concomitante de la pensée néolibérale à travers l'Europe et l'Amérique du Nord. Il n'est pas suffisant, selon eux, de voir la montée de Ronald Reagan et Margaret Thatcher comme des symptômes d'un « retour du marché » et un désengagement de l'État. Au contraire, l'État lui-même est devenu sujet de la rationalité du néolibéralisme durant cette période. Il en est résulté que, à la fin du siècle, l'État n'était plus un rempart contre le néolibéralisme mais un acteur de son inexorable expansion ${ }^{63}$. À la différence de Boltanski, Chiapello et Debray - qui défendent tous, de différentes manières, la capacité de l'État à atténuer les effets dévastateurs du capitalismeDardot et Laval estiment qu'il est un vecteur de diffusion des valeurs néolibérales. Ils affirment par exemple que la rhétorique anticapitaliste du socialisme français sous Mitterrand n'a rien fait pour arrêter la dissémination des « méthodes » néolibérales dans la société française ${ }^{64}$. Et ils soutiennent que la diffusion du gestionnariat dans le secteur public a été néfaste - voire plus - que dans le secteur privé ${ }^{65}$. En résumé, les années 1980 ont inauguré un nouvel âge de rationalité néolibérale irrépressible, qui s'est imposée aux «gouvernants de tous bords comme unique cadre d'intelligibilité des conduites humaines ${ }^{66}$. » Dans ce nouveau monde, l'orientation politique de chacun influe peu : la logique du néolibéralisme s'applique à la droite et la gauche de manière égale. L'idée d'une rationalité néolibérale omniprésente soulève de nombreuses questions - notamment, une question opérationnelle. Alors que Boltanski et Chiapello ont analysé un corpus empirique clair de textes concernant la gestion, et ont insisté sur la relation causale entre ces textes et la pratique de la gestion, La nouvelle raison du monde demeure fortement vague. Dardot et Laval invoquent Foucault pour affirmer que le néolibéralisme est une « stratégie » sans sujet ou agent, ce qui signifie que les origines du néolibéralisme ne sont jamais claires, sauf lorsqu'elles se rapportent à une série de textes politiques et mouvements déconnectés ${ }^{67}$. Pourtant, la conception diffuse du pouvoir qui réside dans l'analyse de Dardot et Laval confirme bien le changement de direction du discours anticapitaliste français, et cela pour tous les problèmes historiques avec une définition 
aussi éphémère que celle du néolibéralisme. Des quatre textes, le pouvoir est le plus clairement décentralisé, éclaté et diffus dans celui-ci. Il y a quelques points communs avec Gorz (qui reçoit une mention favorable à la toute dernière page de son livre), mais peu d'autres penseurs contemporains français et anticapitalistes sont allés aussi loin dans leur décentralisation radicale du pouvoir. Il ne fait pas de doute que l'étonnante absence de réforme après la crise financière de 2008-2009 et l'incapacité ultérieure de la gauche à élaborer une approche critique du capitalisme ont renforcé cette vision. Mais une fois de plus, le capitalisme a survécu à des circonstances peu propices, souvent en collusion avec l'État par des renflouements et des mesures d'austérité. Plus que jamais auparavant, les penseurs anticapitalistes comme Dardot et Laval ont été forcés de prendre en compte le néolibéralisme comme une rationalité omniprésente, dont le pouvoir semblait se trouver nulle part et partout à la fois. Inévitablement, étant donné les difficultés à repérer la capacité et le pouvoir, les critiques potentielles semblent très limitées. Dans d'autres textes et interviews, Dardot et Laval ont demandé la réhabilitation de Marx, la célébration du changement révolutionnaire et les nouvelles formes de « résistance », dans La nouvelle raison du monde, il y a seulement quelques pages en fin d'ouvrage qui discutent des possibilités d'une nouvelle « gouvernementalité ${ }^{68}$. » Cela en fait un texte bien plus pessimiste que ceux de Gorz, Debray et Boltanski et Chiapello. Ni les espaces autonomes, ni les formes originales de solidarité (avec ou sans le soutien de l'État) ne semblent suffire pour briser l'emprise du capitalisme néolibéral. Dans une certaine mesure, ce constat reflète les déceptions répétées de l'extrême gauche : dans une perspective à long terme, la période depuis les années 1970 a été celle d'une domination sans précédent de l'idéologie néolibérale et d'une expansion du capitalisme global. Il est alors logique que les penseurs anticapitalistes auraient dû concentrer leur attention de plus en plus sur les modes de pensée plutôt que sur les relations de production. Si tout le monde est complice du capitalisme - et si nous sommes tous sujets à la « discipline » néolibérale - l'émancipation devra obligatoirement passer avant tout par une libération de la pensée $?^{69}$ C'est un message séduisant, qui a été chaleureusement adopté en France et ailleurs (une traduction anglaise du livre a été publiée par Verso en 2014). Il est encore trop tôt pour dire si les idées de Dardot et Laval auront le même impact sur le long terme que celles des autres penseurs anticapitalistes. Mais leur analyse offre bien un aperçu de la manière dont le discours anticapitaliste pourrait s'accorder avec la persistance d'un système économique et culturel qui était censé disparaître sous le poids de ses propres contradictions.

\section{Quelle critique?}

Les textes étudiés ici offrent bien un aperçu de l'évolution et la variété de l'anticapitalisme français depuis les années 1980. Ils éclairent les manières dont les formes plus anciennes de critiques structurelles, révolutionnaires ou marxistes ont été adaptées pour répondre à une configuration sociopolitique changeante, et hostile. Et ils montrent comment un groupe spécifique d'antilibéraux contemporains a essayé de créer des solutions imaginatives, pragmatiques ou utopiques pour une idéologie néolibérale dominante. 
Les quatre textes ont aussi apporté un éclairage sur les parcours spécifiques que des penseurs français ont adopté afin de dépasser les inquiétudes d'une précédente génération d'intellectuels : Gorz a retravaillé les idées de Sartre ; Debray a essayé d'injecter le zèle révolutionnaire de son tiers-mondisme dépassé dans le républicanisme français ; Boltanski et Chiapello ont affiné la sociologie critique de Bourdieu; et Dardot et Laval ont étendu le travail de Foucault. Cela suggère que la pensée politique française n'a pas simplement vécu une soudaine « disparition de Marx » ou une «mort du sujet » après les critiques antitotalitaires et post-structuralistes de la fin des années 1970 et 1980 . Au contraire, une nouvelle génération de penseurs critiques a été forcée d'élaborer des conceptions plus sophistiquées, décentralisées et fragmentées du pouvoir qui seraient mieux en adéquation avec la réalité de la politique identitaire, les nouveaux mouvements sociaux et la dissolution de la division gauche-droite en France. Dans les années 1970 et 1980, alors que de nombreux instigateurs de la renaissance libérale française demandaient une vision davantage pluraliste de l'histoire française et une meilleure reconnaissance des dangers d'un pouvoir centralisé (qu'il soit « jacobin » ou « totalitaire »), les critiques du capitalisme néolibéral essayaient d'élaborer une vision moins unifiée du pouvoir et de ses multiples manifestations. Cette transformation reflète ce qui se passait pour la politique française en général. Dès les années 1990, il y a eu parmi l'électorat français une impression évidente que le capitalisme néolibéral n'était plus quelque chose « là-bas » aux États-Unis et dans le monde anglo-saxon ${ }^{70}$. Il était désormais une réalité française. Cela a apporté de nouvelles peurs à propos de l'impact potentiel sur l'intervention de l'État et les prestations de l'État. Le signe le plus éclatant de ces peurs a été la vague de grèves qui a balayé la France à l'automne et l'hiver 1995 pour contester la proposition d'Alain Juppé sur la réforme des régimes de retraite du secteur public. L'ampleur des grèves a pris les élites politiques par surprise et a rapidement été interprétée par les commentateurs comme un profond rejet du néolibéralisme - un " grand refus ${ }^{71}$. " Mais pour les jeunes participants, cela a constitué un moment fondateur qui a mené à la formation de nombreux groupes de protestation comme le syndicat étudiant radical SUD (Union syndicale Solidaires) en 1995 et le mouvement antimondialisation ATTAC en 1998. Dès lors, ces organisations ont trouvé des échos dans les mouvements anti-austérité tels que Occupy aux États-Unis, Podemos en Espagne et Syriza en Grèce. 
$\mathrm{N} \cdot \mathrm{O} \cdot \mathrm{T} \cdot \mathrm{E} \cdot \mathrm{S}$

1. Pour différents points de vue sur la montée du néolibéralisme de manière globale, voir Harvey D., A Brief History of Neoliberalism Oxford, Oxford University Press, 2007 ; et Audier S., Néolibéralismes: une archéologie intellectuelle, Paris, Grasset, 2012.

2. Une discussion sur le contexte européen pour ces trois changements est disponible dans Schulz-Forberg H. et Olsen N. (dir.), Re-inventing Western Civilisation : Transnational Reconstructions of Liberalism in Europe in the Twentieth Century, Newcastle, Cambridge Scholars Press, 2014.

3. Les résultats de cette étude sont étudiés dans Perrineau P., « The Great Upheaval: Left and Right in Contemporary French Politics », in France since the 1970s: History, Politics and Memory in an Age of Uncertainty ed. Emile Chabal, London, Bloomsbury Academic, 2014, p. 25-40.

4. À ce propos, voir Chabal E., A Divided Republic : Nation, State and Citizenship in Contemporary France, Cambridge, Cambridge University Press, 2015.

5. Une bonne explication dans Khilnani S., Arguing Revolution: The Intellectual Left in Postwar France, London, Yale University Press, 1993.

6. J'ai été ici influencé par la discussion sur la fragmentation des idées du pouvoir dans la vie intellectuelle américaine dans Rodgers D., Age of Fracture, London, Harvard University Press, 2011, chap. 3 (« The Search for Power »).

7. Son véritable nom était Gérard Horst.

8. Pour différentes critiques positives du travail de Gorz, voir Bowring F., André Gorz and the Sartrean Legacy : Arguments for a Person-Centred Social Theory, Basingstoke, Macmillan, 2000 ; et Fourel C. (dir.), André Gorz: un penseur pour le XXI siècle, Paris, La Découverte, 2012.
9. Par exemple, Gorz A., Stratégie ouvrière et néocapitalisme, Paris, Seuil, 1964.

10. Sur cette période, voir Christofferson M. S., French Intellectuals Against the Left: The Anti-totalitarian Moment of the 1970s, Oxford, Berghahn, 2004.

11. Gorz A., Adieux aux prolétariat : au-delà du socialisme, Paris, Galilée, 1980, p. 214 (« Annexe IIb : vivre sans travailler »). Cette annexe a d'abord été publiée sous le nom de Michel Bosquet, « Quand les chômeurs seront heureux ... », Le Nouvel Observateur, 4 décembre 1978.

12. Lodziak C. et Tatman J., André Gorz : A Critical Introduction, London, Pluto Press, 1997, p. 111.

13. Smith T. B., France in Crisis : Welfare, Inequality and Globalization since 1980, Cambridge, Cambridge University Press, 2004.

14. À propos de cela, par exemple, Gollain F., «André Gorz était-il un écologiste? » Écologie \& politique 1(44) (2012): p. 77-91.

15. Gorz A., Adieux au prolétariat, p. 67.

16. Ibid., p. 91.

17. Ibid., p. 105.

18. Shields J., The Extreme-Right in France: From Pétain to Le Pen, London, Routledge, 2007 ; Perrineau P., La France au front : essai sur l'avenir du Front National, Paris, Fayard, 2014.

19. Gorz A., Adieux au prolétariat, p. 177-178.

20. Voir, par exemple, quelques-uns des essais dans Gorz A., Ecologica, Paris, Galilée, 2008. Ce sont des essais écrits tout au long de sa carrière et sélectionnés par Gorz lui-même peu avant sa mort. Ce livre a été publié à titre posthume.

21. Un bon exemple est Rosanvallon P., L'âge de l'autogestion, ou, La politique au poste de commandement, Paris, Seuil, 1976.

22. L'essai de Debray a d'abord été publié en novembre 1989 dans Le Nouvel Observateur. La version à laquelle je fais référence est « République ou 
Démocratie », in Debray R., Contretemps : éloges des idéaux perdus, Paris, Folio, 1992, p. 15-54.

23. Il y a dorénavant une vaste littérature sur l'affaire du foulard. Pour une sélection, voir Renaut A. et Touraine A., Un débat sur la laïcité, Paris, Stock, 2005 ; Baubérot J., Houziaux A., Bouzar D. et Costa-Lascoux J., Le voile, que cache-t-il ?, Paris, Les Éditions Ouvrières, 2004 ; Wieviorka M. (dir.), Une société fragmentée : le multiculturalisme en débat, Paris, La Découverte, 1996 ; Laborde C., Critical Republicanism : The Hijab Controversy and Political Philosophy, Oxford, Oxford University Press, 2008 ; Bowen J., Why the French Don't Like Headscarves : Islam, the State and the Public Space, Princeton, NJ, Princeton University Press, 2007.

24. Après «République ou Démocratie ", il a écrit deux autres livres sur ce sujet: Debray R., Que vive la République, Paris, Odile Jacob, 1989 et Debray R., La République expliquée à ma fille, Paris, Seuil, 1998.

25. Behrent discute d'autres aspects de la pensée de Debray dans Behrent M. C., «Religion, Republicanism and Depoliticization : Two Intellectual Intineraries Régis Debray and Marcel Gauchet », in Bourg (dir.), After the Deluge, p. 326-349.

26. Debray, « République ou Démocratie », p. 18.

27. Ibid., p. 32, 35.

28. Ibid., p. 26, 34.

29. Ibid., p. 33.

30. Ibid., p. 17.

31. Il y a une critique virulente de l'approche de Debray dans Audier S., La pensée anti-68, Paris, La Découverte, 2010, p. 290-300.

32. Debray, « République ou Démocratie », p. 36-37.

33. Ibid., p. 22.

34. Ibid., p. 46.

35. Régis Debray, «Qu-est-ce que la médiologie », Le Monde Diplomatique, août 1999.

36. Debray, « République ou Démocratie », p. 46.
37. Ibid., p. 52.

38. Parmi d'autres, voir les critiques dans Mounier P. et Jallon H., Les enragés de la République, Paris, La Découverte, 1999 et Lindenberg D., Le rappel à l'ordre : enquête sur les nouveaux réactionnaires, Paris, Seuil, 2002.

39. Finkielkraut A., La défaite de la pensée, Paris, Gallimard, 1987 ; Kriegel B., Philosophie de la République, Paris, Plon, 1998.

40. À ce propos, voir particulièrement Chabal E., « Just Say non? France, Britain and the European Union depuis les années $1980 »$, in Brian J. (dir), National identities in France, London, Transaction Press, 2011, p. 163-186.

41. Boltanski L. et Chiapello E., Le nouvel esprit $d u$ capitalisme, Paris, Gallimard, 2011 [1999].

42. Pour l'élaboration théorique de cette idée de « justification », voir Boltanski L. et Thévenot L., On Justification : Economies of Worth, traduction : Porter C., Princeton, NJ, Princeton University Press, 2006. 43. Boltanski L. et Chiapello E., Le nouvel esprit $d u$ capitalisme, p. 99-166.

44. Ibid., p. 249-464.

45. Ibid., spécialement p. 654-699.

46. Pour un résumé de certains d'entre eux, voir Gay (du) P. et Morgan G. (dir.), New Spirits of Capitalism ? Crises Justifications, and Dynamics, Oxford, Oxford University Press, 2013. Pour une tentative d'applications de certaines des idées sur l'histoire post-guerre de Boltanski et Chiapello, voir les derniers chapitres de Priestland D., Merchant, Soldier, Sage : A New History of Power, London, Penguin, 2013.

47. Boltanski L. et Chiapello E., Le nouvel esprit $d u$ capitalisme, p. 377.

48. Ibid., p. 519.

49. Ibid., p. 550, 694.

50. Ibid., p. 694-695.

51. Ibid., p. 694-695. 
52. Ibid., p. 940-944.

53. «C'est la raison pour laquelle l'espoir qui avait été mis - et qu'ont toujours des gens comme, par exemple, ceux que l'on appelle en France les « républicains 》dans le gentil État qui va protéger le peuple exploité des méfaits du méchant néo-libéralisme, me semble devenu largement illusoire», Duvoux N., «Le pouvoir est de plus en plus savant. Entretien avec Luc Boltanski ».

54. Boltanski L. et Chiapello E., Le nouvel esprit du capitalisme, p. 35 .

55. Cette vision a été le mieux exprimée dans le fameux recueil d'essais dans Furet F., Rosanvallon P., Julliard J., La République du centre : la fin de l'exception française, Paris, Calmann-Lévy, 1988.

56. Dardot P. et Laval C., La nouvelle raison $d u$ monde : essai sur la société néolibérale, Paris, La Découverte, 2009.

57. Behrent M. C., «Liberalism without Humanism : Michel Foucault and the Free-Market Creed, 19761979 », Modern Intellectual History 6 (3), 2009, p. 539-568.

58. Laval C., Jeremy Bentham : le pouvoir des fictions, Paris, Presses Universitaires de France, 1994.

59. Laval C., L'école n'est pas une entreprise : le néolibéralisme à l'assaut de l'enseignement public, Paris, La Découverte, 2004.

60. Sur le groupe Question Marx, voir leur site Internet http://www.questionmarx.typepad.fr/

61. Dardot P. et Laval C., La nouvelle raison $d u$ monde, p. 5.

62. Voir, par exemple, la critique dans Audier, Néolibéralismes.

63. Dardot P. et Laval C., La nouvelle raison du monde, chap. 12 (« Le gouvernement entrepreneurial »).

64. Ibid., p. 327.

65. Ibid., p. 431-433.

66. Ibid., p. 277.
67. Ibid., p. 276.

68. Leur dernier ouvrage propose une plateforme pour le changement démocratique et révolutionnaire. Dardot P. et Laval C., Commun : Essai sur la révolution au XXI siècle, Paris, La Découverte, 2014.

69. Dardot P. et Laval C., La nouvelle raison du monde, p. 300-301.

70. À ce propos, voir Chabal E., « The Rise of the Anglo-Saxon : French Perceptions of Anglo-American World in the Long Twentieth Century ", French Politics, Culture and Society 31 (1), 2013, p. 24-46 et Jennings J., « France and the Anglo-Saxon Model : Contemporary and Historical Perspectives », European Review 14 (4), 2006, p. 537-554.

71. Touraine A. et al., Le grand refus : réflexions sur la grève de décembre 1995, Paris, Fayard, 1996. 


\section{$R \cdot E ́ S \cdot U \cdot M \cdot E ́$}

Malgré une transformation en profondeur du paysage politique depuis les années 1980, les français restent particulièrement hostiles au libéralisme. Dans cet article, je tente d'élucider ce paradoxe en analysant une des formes d'antilibéralisme les plus connues : l'anticapitalisme de gauche. À travers quatre textes clés - Adieux au prolétariat (1980) d'André Gorz, « République et démocratie » (1989) de Régis Debray, Le nouvel esprit du capitalisme (1999) de Luc Boltanski et Ève Chiapello et La nouvelle raison du monde (2010) de Pierre Dardot et Christian Laval - je trace l'émergence d'un nouveau discours anticapitaliste qui s'appuie désormais sur des concepts tel que « l'esprit » et « la raison », plutôt que sur des idées de « classe » ou de « révolution ». On découvre alors, que même si la pensée anticapitaliste de gauche critique explicitement le capitalisme néolibéral du $\mathrm{XXI}^{\mathrm{e}}$ siècle, elle s'est aussi adaptée à la diffusion et l'émiettement du pouvoir dans un monde néolibéral.

\begin{abstract}
Despite a profound transformation in the ideological landscape since the 1980s, the French remain unusually hostile to liberalism. In this article, I try to explain why this is the case by looking at one of the dominant forms of anti-liberalism in France: left-wing anti-capitalism. Through a discussion of four key texts -André Gorz's Adieux au prolétariat (1980), Régis Debray's "République et démocratie" (1989), Luc Boltanski and Ève Chiapello's Le nouvel esprit du capitalisme (1999), and Pierre Dardot and Christian Laval's La nouvelle raison du monde (2010) - I trace the shift in left-wing anti-capitalist thought from a language of 'class' and 'revolution', to one of 'spirit' and 'reason'. I suggest that, while left-wing anti-capitalist thought has sys-
\end{abstract}

tematically attacked the foundations of 21 st century neo-liberal capitalism, it has nevertheless been forced to adapt to a more decentered and diffuse notion of power in a neo-liberal world. 